\title{
Physical exercise intervention at school improved hepcidin, inflammation, and iron metabolism in overweight and obese children and adolescents
}

\author{
Susana Coimbra ${ }^{1}$, Cristina Catarino², Henrique Nascimento ${ }^{2}$, Ana Inês Alves ${ }^{3}$, Ana Filipa Medeiros ${ }^{3}$, Elsa Bronze-da-Rocha ${ }^{2}$, \\ Elísio Costa ${ }^{2}$, Petronila Rocha-Pereira ${ }^{4}$, Luísa Aires ${ }^{3}$, André Seabra ${ }^{3}$, Jorge Mota ${ }^{3}$, Helena Ferreira Mansilha ${ }^{5}$, Carla Rêgo ${ }^{6}$, \\ Alice Santos-Silva ${ }^{2}$ and Luís Belo ${ }^{2}$
}

BACKGROUND: Obesity is often associated with iron deficiency in children and adolescents. We aimed to study the effect of an 8-month physical exercise (PE) intervention on hepcidin and other markers of inflammation and on iron status in overweight/obese children and adolescents.

METHODS: Seventy-three overweight/obese children and adolescents participated in the 8-month-long longitudinal study. They were divided into two groups according to their participation in an after-school PE program: the PE group $(n=44)$ and the control group $(n=29)$. Hepcidin, interleukin (IL)-6, C-reactive protein (CRP), iron, ferritin, transferrin, and soluble transferrin receptor (sTfR) were evaluated.

RESULTS: At baseline, IL-6 correlated positively with hepcidin and negatively with iron and transferrin saturation, suggesting that increasing adiposity associates with increasing IL-6 and hepcidin synthesis, reducing iron availability. After 8 months, the PE group showed a decrease in BMI $Z$-score $(P=0.003)$, body fat mass $(P=0.012)$, CRP $(P=0.002)$, IL-6 $(P=0.048)$, ferritin ( $P=0.013)$, hepcidin $(P=0.040)$, and sTfR $(P=0.010)$, and an increase in iron concentration $(P=0.002)$. Moreover, the $P E$ group, when compared with the control group, showed lower weight $(P=0.026), \mathrm{BMI} \quad(P=0.040)$, waist circumference $(P=0.010)$, and waist-to-height ratio $(P=0.046)$. CONCLUSION: We showed that an 8-month-long intervention at school allowed a reduction in BMl z-score and an improvement in inflammation, reducing hepcidin levels and the disturbances in iron status.

O besity is a critical public health problem and its prevalence has increased significantly over the past three decades. Iron deficiency is one of the most predominant worldwide nutritional problems, being prevalent in both developing and industrialized countries (1). Several studies have confirmed an association between obesity and hypoferremia in adult men, women, and post-menopausal women, as well as in children and adolescents (2-8). Obesity is considered a potential independent risk factor for developing iron deficiency $(3,4,7,9)$. Different mechanisms have been proposed to explain the association between obesity and iron deficiency (10), and the most widely accepted is that hypoferremia is a consequence of the chronic low-grade inflammation state that characterizes obesity (11). Inflammation enhances hepcidin production, the major regulator of iron availability for erythropoiesis. Hepcidin is predominantly expressed in the liver, but it can be also expressed in other tissues, such as the heart, kidney, adipose tissue (also an adipokine), pancreas, and hematopoietic cells (12). In inflammatory conditions, its concentration increases, triggering endocytosis and proteolysis of ferroportin. Thus, the efflux of ferrous iron from major iron-transporting tissues-namely, duodenal enterocytes, iron-recycling macrophages, and iron-storing hepatocytes-into plasma is reduced, and the iron accumulates in their cytoplasm as ferritin (13). The regulation of hepcidin by inflammation occurs in response to pro-inflammatory cytokines, such as interleukin (IL)-6 (Figure 1) (14-16).

In inflammatory processes, soluble transferrin receptor (sTfR) is considered a more reliable and sensitive marker of iron status compared with ferritin, which is also an acutephase protein (17). Thus, the association of sTfR and hepcidin in the study of iron metabolism probably provides a more valuable information when assessing iron status under inflammatory conditions.

In children with high BMI $z$-score, the supplementation with iron has been proven to be of little efficacy (18), suggesting that iron supplementation is not the best way to improve iron status in these patients. Physical activity and aerobic exercise, and/or adequate changes in nutritional behavior, are considered reliable treatment options for obesity. Indeed, aerobic exercise appears to neutralize the inflammatory state, contributing to 


\section{Articles $\mid$ Coimbra et al.}

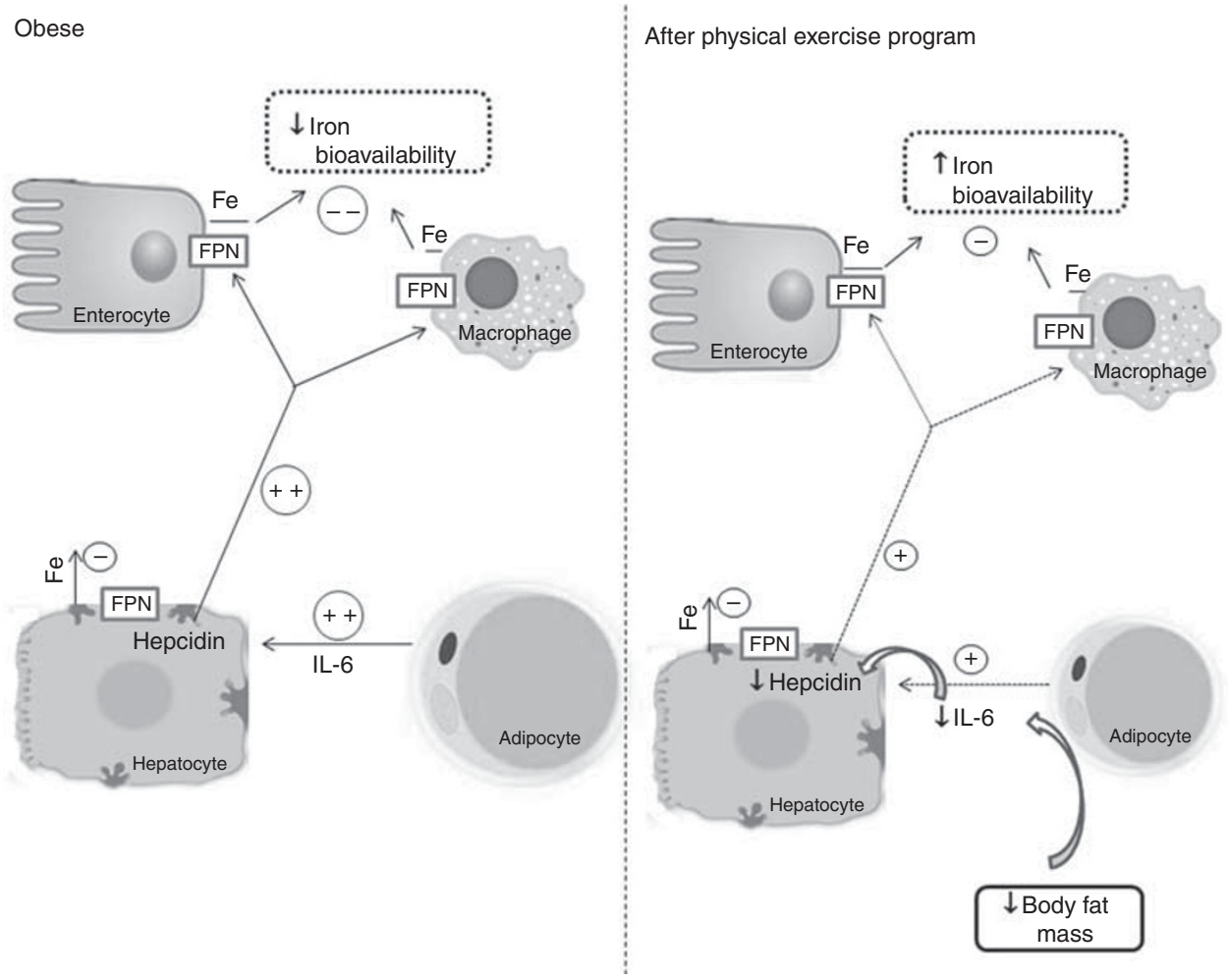

Figure 1. Schematic diagram representative of the interplay between inflammation, hepcidin, and iron bioavailability in obesity and after a physical exercise program. In obese and overweight children and adolescents, the hepatic production of hepcidin is enhanced by inflammation, namely by interleukin (IL)-6, produced by adipocytes; hepcidin induces ferroportin (FPN) degradation at the membrane surface of enterocytes, hepatocytes, and macrophages, inhibiting iron ( $\mathrm{Fe}$ ) absorption and mobilization from iron stores, thus reducing iron bioavailability for erythropoiesis; after an 8-monthlong exercise program, a reduction in body fat mass was associated with a decrease in IL-6, leading to a reduction in hepcidin that favors iron absorption and mobilization, increasing iron availability.

improving insulin sensitivity and lipid profile (19). A 6-monthlong intervention program including 15 obese children (without a control group) that combined a nutritionally balanced diet with lifestyle modifications induced a BMI decrease that was associated with hepcidin reduction, and, although no alteration was found for iron status markers, iron absorption was improved (20). In agreement, a 1-year-long weight-loss program performed in overweight and obese children reported an improvement in markers of inflammation and iron status; in this study hepcidin levels were not evaluated (21). To better understand the effects of continuous physical exercise (PE) interventions in the relationship between hepcidin, inflammation, and iron availability in obese and overweight children, larger studies evaluating hepcidin and other markers of inflammation, and iron metabolism, are needed.

The aim of this study was to clarify how obesity-associated inflammation can disturb iron metabolism in overweight and obese children and adolescents, and study the effect of an 8month-long PE intervention program at school on their levels of hepcidin and other markers of inflammation and on iron metabolism.

\section{METHODS}

\section{Subjects}

This study is included in a larger school-based project that aimed to evaluate the impact of lifestyle intervention on obesity-related comorbidities in children and adolescents (22). Overweight and obese children and adolescents participated in the study, after obtaining informed and written consent from their parents. Two recruitment strategies were used. A part of the population was identified from medical records from two outpatient clinics of pediatric obesity in Oporto and invited to participate during the medical appointments (with the presence of children/adolescents and their parents). A second group of children and adolescents were recruited from public schools from an Oporto suburban setting. In this case, the project was presented to parents and opened to all students meeting the inclusion criteria, explaining that it was directed more towards overweight and obese children and adolescents.

The inclusion criteria were as follows: age 5-17 years, being overweight or obese (BMI $>1$ SD or BMI $>2$ SDs above age-specific and sex- specific World Health Organization (WHO) reference median values, respectively) (23), having sedentary behavior (absence of regular physical activity-less than $2 \mathrm{~h}$ per week), and absence of any medical condition that could affect the study results or limit physical activity. Children and adolescents who were participating in any other PE, nutrition, and/or weight-loss programs were excluded, as well as smokers, subjects with diabetes mellitus, endocrine 


\section{Inflammation and iron in obese children $\quad$ Articles}

disorders, or inflammatory or infectious diseases, and subjects under medication that could interfere with our results.

All eligible participants were invited to participate in a schoolbased PE promotion program carried out in 5 primary and 2 middle and high public schools from an Oporto suburban setting. The intervention study consisted of an 8-month-long PE program (corresponding to a school-year period) that took place in the aforementioned schools. Only those participants who had a compliance of at least $75 \%$ in the PE program, and had undergone blood analytical studies at the beginning and at the end of the study were included in the final analysis.

Forty-four of the seventy-three individuals enrolled in the study agreed to participate in the PE program. Twenty-nine subjects, who accepted to participate in the study, but did not want to participate in the PE program, were included in the control (C) group.

The study protocol was approved by the Committee on Ethics of both Pediatric Clinic and the Review Committee of the Scientific Board of the Faculty of Sport of the University of Porto. The Regional Education Board approved the study protocol, and students, parents, and schools agreed to participate. The nature, benefits, and risks of the study were explained to the volunteers.

\section{Anthropometric Characterization and Clinical Evaluation}

Height and weight were measured with participants wearing shorts and t-shirts. Height was measured using a fixed stadiometer (Holtain, Crymych, UK). Weight was measured with the scale Tanita MC 180 MA MA (Tanita, Amsterdam, The Netherlands). BMI was also calculated $\left(\mathrm{kg} / \mathrm{m}^{2}\right)$. $z$-score values for BMI were obtained with WHO AnthroPlus software, which is available in the WHO website (http://www.who.int/growthref/tools/en/).

Waist circumference was measured with a metallic tape (Holtain) at the superior border of the iliac crest, according to the protocol of the National Health and Nutrition Examination Survey (NHANES), and the ratio between waist circumference and height was calculated.

Body composition was evaluated using dual-energy X-ray absorptiometry (Hologic QDR $4500 \mathrm{~A}$, Hologic, Waltham, MA). This unit was calibrated according to manufacturer's instructions, and a trained technician conducted all examinations. The percentage of body fat mass was measured.

Biological maturation was clinically assessed on the basis of Tanner stages (24).

\section{Physical Activity Intervention Program}

Participants were integrated into the "Acorda Project", a schoolbased intervention program focused on young people with overweight and obesity that aimed to change behaviors by providing easy access to PE associated with food counseling and clinical supervision. The participants were involved in the PE program over a period of 8 months, from September to May. Besides regular classes of PE at school 3 times a week, participants were enrolled in an extra-activity PE program twice a week, resulting in a total of $5 \mathrm{~h}$ per week (measured under school/project supervisors) of moderate to vigorous $\mathrm{PE}$, matching the international recommendations (25-27). The PE proposed by the program aimed to increase moderate to vigorous $\mathrm{PE}$ intensities. All activities were performed in the schools' indoor sports facilities and were taught by physical education teachers after regular school classes. Exercises and games were progressively intensified as individually tolerated. Training intensity and compliance between individuals was defined to induce heart rate higher than $80 \%$ of each child's maximum heart rate. To ensure this, 10 randomly selected participants used a portable heart rate monitor (Polar Team2 Pro, Polar, Finland) and an accelerometer (MTI, model GTX3, as described below) during sessions. The average number of sessions attended (adherence) was $>85 \%$ for the PE group.

\section{Blood Samples}

Blood was collected for laboratory analysis after an overnight fast and after clinical examination at the research centers, before and after the 8-month period. Blood was obtained by venepuncture, collected into EDTA-containing tubes, and processed within $2 \mathrm{~h}$ of collection. Aliquots of plasma were prepared and immediately stored at $-80^{\circ} \mathrm{C}$ until assayed.

\section{Assays}

Plasma levels of hepcidin, sTfR, and IL-6 were evaluated by enzyme immunoassays (Hepcidin-25, Bachem Group, Peninsula Laboratories, San Carlos, CA; human sTfR immunoassay, R\&D Systems, Minneapolis, MN; and Human IL-6 BMS213HS, Bender MedSystems, Vienna, Austria). Iron concentration was determined following a colorimetric method (Iron, Randox Laboratories, North Ireland, $\mathrm{UK})$, whereas ferritin, transferrin, and C-reactive protein (CRP) were measured with immunoturbidimetry (Ferritin, Randox Laboratories; Transferrin, Randox Laboratories ; and CRP (latex) High-Sensitivity, Roche Diagnostics, Basel, Switzerland). Transferrin saturation (TS) was calculated according to the formula TS $(\%)=70.9 \times$ serum iron levels $(\mu \mathrm{g} / \mathrm{dl}) /$ transferrin $(\mathrm{mg} / \mathrm{dl})$. Red blood cell count, hemoglobin concentration, hematocrit, mean corpuscular volume, mean corpuscular hemoglobin, and mean corpuscular hemoglobin concentration were evaluated by using an automatic blood cell counter (Sysmex XT-1800i; Sysmex, Hamburg, Germany).

\section{Statistical Analysis}

Statistical analysis was performed using the Statistical Package for Social Sciences (SPSS, version 22.0, Chicago, IL) for Windows. Kolmogorov-Smirnov analysis was used to test whether the results were normally distributed. The parametric variables are presented as mean $\pm S D$ and the non-parametric variables are presented as median (interquartile range). Differences between groups at baseline were tested using the chi-squared test and Fisher's exact test for categorical variables; for continuous variables, the unpaired Student's $t$-test or the Mann-Whitney $\mathrm{U}$ test was used, according to the distribution of the variable. Within-group changes over the 8-month intervention period were evaluated by Wilcoxon signed ranks test and by the paired Student's $t$-test, in accordance with the Gaussian distribution of the variables. Spearman's rank correlation coefficient was used to evaluate relationships between sets of data. A $P$ value lower than 0.05 was considered statistically significant.

\section{RESULTS}

The anthropometric evaluations observed for the PE and the $\mathrm{C}$ group are presented in Table 1. At baseline, the two groups were matched for BMI, BMI $z$-score, waist-to-height ratio, and percentage of body fat mass, as well as for age, gender, and Tanner stage (76 and $83 \%$ with Tanner stage $\geq 2$ in PE and control groups, respectively). The PE group showed lower iron and TS values compared with the C group (Table 1).

At the end of the study, the $\mathrm{C}$ group showed a significant increase in body fat mass percentage (Table 1). The overweight and obese children who underwent PE intervention presented a significant decrease in BMI $z$-score and body fat mass (Table 1). These alterations were accompanied by a significant decrease in the levels of CRP, IL-6, ferritin, hepcidin, and sTfR, and an increase in iron concentration and TS; a significant increase in mean corpuscular volume was also observed (Table 1).

After the 8-month period, the PE group, compared with the $\mathrm{C}$ group, showed lower values of waist-to-height ratio (Table 1).

At baseline, when considering all the 73 subjects, BMI $z$-score correlated significantly and positively with sTfR $(r=0.292, P=0.012)$ and transferrin $(r=0.390, P=0.001)$; hepcidin correlated positively and significantly with IL-6 


\section{Articles | Coimbra et al.}

Table 1. Anthropometric and analytical data in overweight (OW) and obese (OB) children and adolescents before (T1) and after an 8-monthlong exercise program (T2), for the PE and control (C) groups

\begin{tabular}{|c|c|c|c|c|c|c|c|c|}
\hline & \multirow{2}{*}{$\frac{C \text { group }}{\mathrm{T} 1}$} & \multicolumn{2}{|c|}{$(n=29 ; 26$ OB +3 OW $)$} & \multirow{2}{*}{$\begin{array}{c}\text { PE group } \\
\text { T1 }\end{array}$} & \multicolumn{2}{|c|}{$(n=44 ; 35 \mathrm{OB}+9$ OW $)$} & \multirow{2}{*}{$\frac{P(C \text { vs. } \mathrm{PE})}{\mathrm{T} 1}$} & \multirow{2}{*}{$\frac{P(C \text { vs. } P E)}{\mathrm{T} 2}$} \\
\hline & & $\mathrm{T} 2$ & $P($ T1vs.T2) & & $\mathrm{T} 2$ & $P($ T1vs.T2) & & \\
\hline \multicolumn{9}{|l|}{ Anthropometric data } \\
\hline Age (years) & $10.3(9.1-11.6)$ & $10.7(9.5-12.1)$ & $\leq 0.001$ & $9.1(7.8-11.7)$ & $9.6(8.4-12.4)$ & $\leq 0.001$ & 0.083 & 0.092 \\
\hline Gender (\%F/\%M) & $52 / 48$ & $52 / 48$ & - & $45 / 55$ & $45 / 55$ & - & 0.420 & - \\
\hline \multicolumn{9}{|c|}{ Tanner stage $(\% \mathrm{~F} / \% \mathrm{M})$} \\
\hline Stage 1 & $3.45 / 13.79$ & $3.45 / 6.90$ & - & $9.09 / 13.64$ & $9.09 / 6.82$ & - & $0.816^{a}$ & $0.512^{\mathrm{a}}$ \\
\hline Stage 2 & $24.14 / 13.79$ & $3.45 / 20.69$ & & $18.18 / 15.91$ & $6.82 / 18.18$ & & & \\
\hline Stage 3 & $17.24 / 13.79$ & $37.93 / 13.79$ & & $11.36 / 11.36$ & $18.18 / 13.64$ & & & \\
\hline Stage 4 & $6.90 / 3.45$ & $6.90 / 3.45$ & & $6.82 / 11.36$ & $9.09 / 9.09$ & & & \\
\hline Stage 5 & $3.45 / 0.00$ & $3.45 / 0.00$ & & $0.00 / 2.27$ & $2.27 / 6.82$ & & & \\
\hline Height $(\mathrm{cm})$ & $144(138-158)$ & $146(140-158)$ & $\leq 0.001$ & 139 (130-151) & $142(132-155)$ & $\leq 0.001$ & 0.030 & 0.106 \\
\hline Weight $(\mathrm{kg})$ & $54.0(41.3-61.7)$ & $55.8(44.9-66.0)$ & 0.002 & $43.7(33.6-60.5)$ & $44.6(36.7-63.3)$ & $\leq 0.001$ & 0.045 & 0.026 \\
\hline BMI $\left(\mathrm{kg} / \mathrm{m}^{2}\right)$ & $25.4 \pm 3.5$ & $25.8 \pm 3.7$ & 0.053 & $24.1 \pm 4.8$ & $23.8 \pm 4.7$ & 0.152 & 0.204 & 0.040 \\
\hline BMI z-score & $2.46(2.24-2.95)$ & $2.50(2.15-2.80)$ & 0.210 & $2.33(2.03-3.04)$ & $2.10(1.80-2.90)$ & 0.003 & 0.297 & 0.070 \\
\hline WC (cm) & $82.5(73.7-93.8)$ & $86.5(78.7-95.0)$ & 0.216 & $76.0(69.6-86.9)$ & $78.6(70.0-87.2)$ & 0.156 & 0.030 & 0.010 \\
\hline $\mathrm{WtH}$ & $0.58 \pm 0.05$ & $0.58 \pm 0.05$ & 0.366 & $0.56 \pm 0.07$ & $0.56 \pm 0.06$ & 0.806 & 0.256 & 0.046 \\
\hline Body fat mass (\%) & $38.7(34.2-41.5)$ & $40.4(36.8-44.7)$ & 0.009 & $40.1(35.1-43.7)$ & $39.5(33.8-42.6)$ & 0.012 & 0.250 & 0.182 \\
\hline \multicolumn{9}{|l|}{ Hematologic data } \\
\hline $\mathrm{RBC}\left(\times 10^{12} / \mathrm{l}\right)$ & $4.96 \pm 0.32$ & $4.93 \pm 0.36$ & 0.530 & $4.89 \pm 0.29$ & $4.89 \pm 0.30$ & 0.939 & 0.332 & 0.539 \\
\hline $\mathrm{Hb}(\mathrm{g} / \mathrm{dl})$ & $13.7 \pm 0.6$ & $13.6 \pm 0.7$ & 0.561 & $13.5 \pm 0.9$ & $13.6 \pm 0.8$ & 0.223 & 0.335 & 0.941 \\
\hline Hct (\%) & $41.3 \pm 1.89$ & $41.1 \pm 2.6$ & 0.671 & $40.4 \pm 2.8$ & $41.0 \pm 3.0$ & 0.166 & 0.115 & 0.809 \\
\hline MCV (fl) & $84.0(80.5-86.0)$ & $83.0(82.0-85.5)$ & 0.971 & $83.0(80.0-85.6)$ & $84.0(82.0-86.0)$ & 0.033 & 0.342 & 0.522 \\
\hline $\mathrm{MCH}(\mathrm{pg})$ & $27.7 \pm 1.4$ & $27.7 \pm 1.4$ & 0.947 & $27.7 \pm 1.4$ & $27.9 \pm 1.3$ & 0.078 & 0.991 & 0.443 \\
\hline $\mathrm{MCHC}(\mathrm{g} / \mathrm{dl})$ & $33.0(32.6-33.4)$ & $32.8(32.5-33.9)$ & 0.707 & $33.5(32.6-34.2)$ & $33.0(32.3-33.9)$ & 0.294 & 0.245 & 0.866 \\
\hline \multicolumn{9}{|c|}{ Inflammatory and iron status parameters } \\
\hline $\mathrm{CRP}(\mathrm{mg} / \mathrm{l})$ & $0.98(0.40-2.22)$ & $1.10(0.28-1.61)$ & 0.888 & $0.86(0.21-3.69)$ & $0.61(0.21-1.70)$ & 0.002 & 0.701 & 0.596 \\
\hline IL-6 (pg/ml) & $0.64(0.40-0.99)$ & $0.54(0.42-1.03)$ & 0.837 & $0.86(0.53-1.70)$ & $0.72(0.42-1.06)$ & 0.048 & 0.062 & 0.765 \\
\hline Hepcidin (ng/ml) & $20.4(14.2-31.3)$ & $20.7(14.7-29.9)$ & 0.567 & $22.8(12.2-34.2)$ & $17.8(10.8-26.2)$ & 0.040 & 0.978 & 0.219 \\
\hline Iron $(\mu \mathrm{g} / \mathrm{dl})$ & $41.0(32.5-49.5)$ & $32.0(25.5-49.5)$ & 0.078 & $31.5(22.5-40.8)$ & $40.0(31.0-56.0)$ & 0.002 & 0.021 & 0.200 \\
\hline sTfR (nmol/l) & $24.6 \pm 7.5$ & $24.6 \pm 6.3$ & 0.977 & $23.9 \pm 4.6$ & $22.3 \pm 5.2$ & 0.010 & 0.643 & 0.107 \\
\hline Transferrin (mg/dl) & $279(258-310)$ & $290(262-305)$ & 0.682 & $278(255-322)$ & $271(243-301)$ & 0.238 & 0.782 & 0.165 \\
\hline TS (\%) & $9.88(7.74-12.78)$ & $8.89(6.50-12.52)$ & 0.127 & 7.81(5.85-10.72) & $10.06(7.90-14.09)$ & 0.002 & 0.031 & 0.099 \\
\hline Ferritin (ng/ml) & $49.1(39.1-69.7)$ & $48.4(31.4-66.3)$ & 0.729 & $49.2(30.3-79.6)$ & $45.7(29.9-65.5)$ & 0.013 & 0.752 & 0.569 \\
\hline
\end{tabular}

BMI, body mass index; CRP, C-reactive protein; F, female; Hb, hemoglobin; Hct, hematocrit; IL, interleukin; M, male; MCH, mean cell hemoglobin; MCHC, mean cell hemoglobin concentration; MCV, mean cell volume; PE, physical exercise group; RBC, red blood cell; sTfR, soluble transferrin receptor; TS, transferrin saturation; WC, waist circumference; WtH, waist-to-height ratio. Results are presented as mean \pm SD or as median (interquartile range). Statistically significant values are highlighted using bold numbers.

aWen considering and comparing the two groups - only female gender groups or only male gender groups - the Tanner stage persisted without statistical significance ( $P=0.795$ and $P=0.881$, at $\mathrm{T} 1$, and $P=0.539$ and $P=0.747$, at $\mathrm{T} 2$, respectively).

(Figure 2) and with ferritin $(r=0.478, P<0.001)$; IL-6 correlated negatively with iron $(r=-0.237, P=0.045)$ and TS $(r=-0.250, P=0.034)$; and CRP correlated positively with IL-6 $(r=0.308, P=0.006)$ and with ferritin $(r=0.237$, $P=0.036)$.

After the 8-month period, the correlation of hepcidin with IL-6 and that of BMI $z$-score with sTfR were still observed in the C group $(r=0.416, \quad P=0.025 ; \quad r=0.445, \quad P=0.016$; respectively) but not in the $\mathrm{PE}$ group. Hepcidin correlation with ferritin persisted in the $\mathrm{PE}$ and $\mathrm{C}$ groups (Figure 3).

In the PE group, the percentual change $(\% \Delta)$, from baseline to 8 months of intervention, of hepcidin correlated significantly and positively with the $\% \Delta$ of IL-6 (Figure 4 ) and $\% \Delta$ of ferritin $(r=0.310, P=0.043)$; the $\% \Delta$ of IL-6 correlated 


\section{Inflammation and iron in obese children Articles}

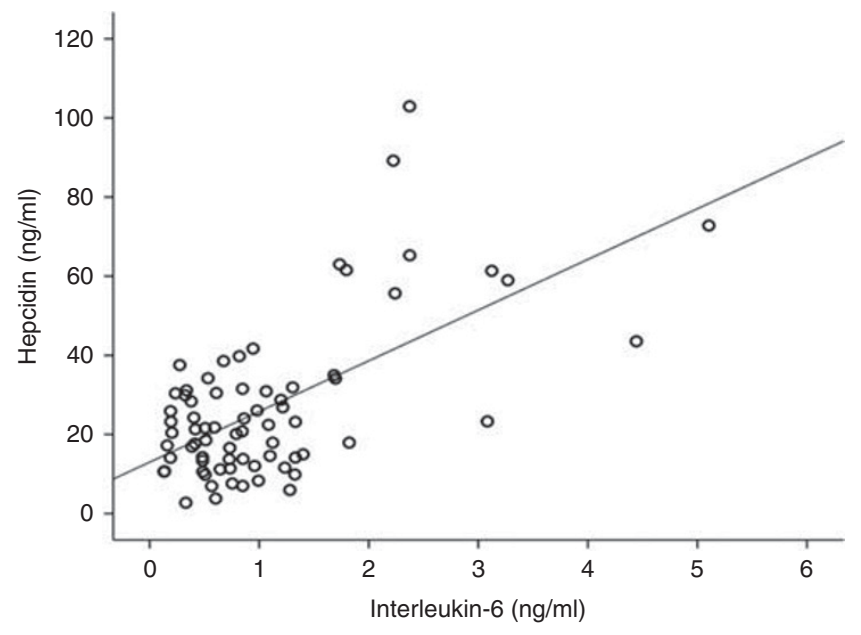

Figure 2. Correlations observed at baseline between circulating levels of hepcidin and interleukin-6 $(n=73 ; r=0.398 ; P=0.001)$ in overweight and obese children and adolescents.

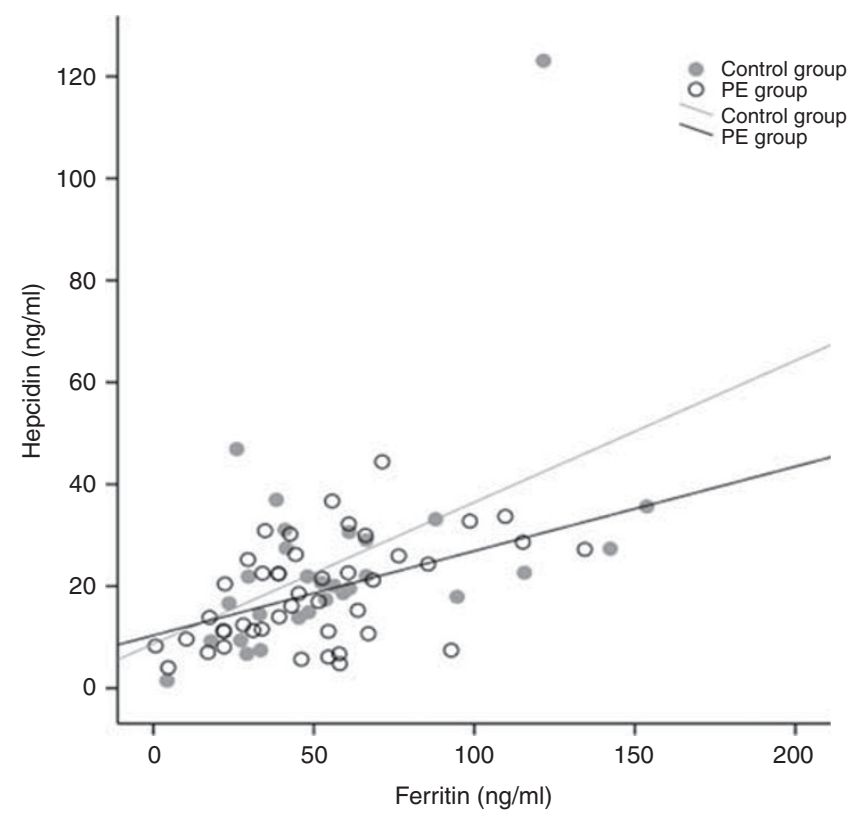

Figure 3. Correlations observed between hepcidin and ferritin after an 8-month-long exercise program in the physical exercise ( $\mathrm{PE} ; n=44$; $r=0.459 ; P=0.002)$ and control groups $(n=29 ; r=0.525 ; P=0.003)$.

significantly and positively with the $\% \Delta$ of CRP $(r=0.583$, $P<0.001)$ and negatively with the $\% \Delta$ of iron $(r=-0.336$, $P=0.032)$. In the $C$ group, the $\% \Delta$ of hepcidin correlated significantly and negatively with the $\% \Delta$ of TS $(r=-0.423$, $P=0.022)$.

\section{DISCUSSION}

The improvement in iron status is important for several biological functions, such as hematological and cognitive functions. Indeed, it is widely accepted that iron deficiency has a negative impact on cognition and behavior (28), and

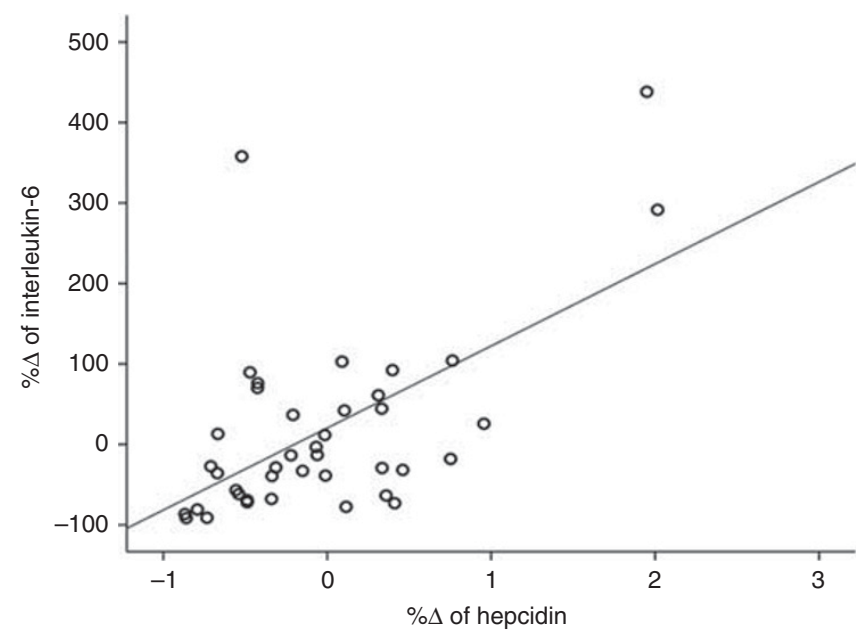

Figure 4. Correlations observed in the physical exercise group of the percentual change $(\% \Delta)$, from baseline to 8 months of intervention, of interleukin- 6 with $\% \Delta$ of hepcidin $(r=0.465, P=0.002)$.

thus an adequate iron availability improves these functions. Epidemiological data have shown that disturbances in iron metabolism are common in obese children and adolescents, presenting usually low plasma iron, despite increased ferritin levels $(2,5-7,29)$. Few hypotheses have been proposed to explain this association: namely, an inadequate dietary iron ingestion, an increase in iron requirements, a consequence of a higher body mass and an increase in blood volume, as well as a poor iron absorption $(2,5,6)$. Some studies performed in children and adolescents showed that inadequate iron ingestion per se does not explain this relationship $(7,30,31)$. Obesity has also been associated with low-grade inflammation that triggers the production of hepcidin, which is mainly produced by the liver, but it might be also produced by adipose tissue. In fact, few studies have reported that hepcidin levels are higher in obese children as compared with normal weight children (29-31). Hepcidin, by inducing ferroportin degradation, inhibits iron absorption and mobilization from iron stores, reducing circulating iron and increasing iron stores. Hepcidin expression is induced by iron overload and inflammation and is suppressed by hypoxia, iron deficiency, and ineffective erythropoiesis $(32,33)$. During inflammatory conditions, like obesity, the expression of hepcidin is mediated through the IL-6/STAT3 (signal transducer and activator of transcription-3) pathway (15).

To further clarify the disturbances in iron metabolism and its association with inflammation in the pediatric obese population, we conducted an evaluation of inflammatory markers and iron metabolism in a sub-sample of subjects from the Acorda Project, as mentioned earlier. As both transferrin and ferritin levels are known to be altered in inflammation, we also evaluated sTfR, as it is not influenced by inflammation (17). We observed a positive correlation at baseline between sTfR and BMI $z$-score that corroborates the association between obesity and iron deficiency (2-7). In fact, it is well known that the levels of sTfR increase with 


\section{Articles | Coimbra et al.}

erythropoietic activity and with the increase in iron requirements, reflecting an accelerated erythropoiesis and/or an inadequate iron availability for erythropoiesis. We also found a positive correlation between hepcidin and ferritin at baseline and after the intervention in both groups. Hepcidin induces iron accumulation in the cytoplasm of duodenal enterocytes, macrophages, and hepatocytes in the form of ferritin (13), explaining this correlation.

Our results also show a positive correlation between IL-6 and hepcidin and an inverse correlation of IL- 6 with iron and TS, showing the role of IL-6 in the modulation of hepcidin and iron metabolism. Indeed, those participants who achieved more substantial reductions in IL-6 also presented more pronounced reductions in hepcidin (Figure 4), showing that the increase in the inflammatory mediator IL-6 enhances hepcidin production, favoring a functional iron deficiency in obesity (Figure 1).

The adipose tissue is an important source of IL-6 and also expresses hepcidin (34). The adipocytes seem to be able to produce hepcidin in response to an inflammatory stimuli, such as IL-6 (34). This may justify our results, as the correlation of hepcidin with IL-6 and of BMI $z$-score with sTfR remained significant only in the $C$ group, which showed a significant increase in body fat mass and no decrease in BMI $z$-score. It has been hypothesized that hepcidin secreted by increased adipose tissue during obesity may contribute significantly to the circulating levels of hepcidin, disturbing iron metabolism and erythropoiesis(10). This may explain the loss of correlations observed after BMI $z$-score reduction in the PE group, and supports the hypothesis that in the context of obesity IL-6 and hepcidin may exert a major effect in modulating iron metabolism (Figure 1). Actually, the lack of correlations in the PE group after intervention may express a more homogenous group of individuals at the end of study.

In the PE group, the physical intervention led to a decrease in BMI $z$-score and body fat mass. The reduction in adipose mass was associated with a decrease in inflammation, as shown by the significant reduction in IL-6, CRP, and ferritin levels. Strengthening the role of inflammation in the disturbances in iron metabolism during obesity, we found that the decrease in IL-6 led to a reduction in hepcidin levels, allowing an improvement in iron availability (Figure 1). Indeed, circulating levels of iron and TS increased, showing an improvement in iron absorption and mobilization, and sTfR levels decreased, showing an improvement in iron availability for erythropoiesis. None of these alterations were observed in children from the $\mathrm{C}$ group, in whom an enhancement in body fat mass was registered. Our data show that aerobic exercise practice is an efficient way to reduce adiposity, improving iron profile, in obese children.

There are only a few studies addressing the cross-talk between obesity, inflammation, hepcidin, and iron metabolism in children and adolescents, and the results are controversial, probably due to the variety in study designs (e.g. number of obese individuals, type of intervention, and duration of follow-up). In accordance with our data, Amato et al. (20) reported a decrease in hepcidin and an increase in iron absorption, following a reduction in BMI. Gong et al. (21) also reported that weight loss, achieved by overweight and obese children through a 1-year program, was associated with an improvement in iron status and inflammatory markers. Studies performed in adults also reported an association between fat loss, reduction in inflammation and hepcidin, and improvement in iron profile $(35,36)$.

As the main goal of this work was to evaluate the impact of PE on the several parameters under study, children and their parents chose the group to be included, either the $\mathrm{PE}$ or the $\mathrm{C}$ group. We found that at baseline the $\mathrm{C}$ group presented significantly higher iron and TS. The level of circulating iron increases during childhood, being associated with physiological changes, such as growth and hormonal changes. In the present study, the $\mathrm{C}$ and $\mathrm{PE}$ groups were equilibrated for age, Tanner stage, and BMI $z$-score, but the $\mathrm{C}$ group presented higher values for height and weight at baseline. A more rapid growth of individuals in the $\mathrm{C}$ group may justify, at least in part, the higher iron levels and TS values, though a contribution of environmental factors (e.g. diet) cannot be excluded.

At the end of the PE intervention program, when comparing the $\mathrm{C}$ and $\mathrm{PE}$ groups, in spite of the decrease in $\mathrm{BMI}$ in the $\mathrm{PE}$ group, we did not find significant differences in the studied analytical markers. A more intense or longer physical intervention program or a program that combines physical activity with nutrition intervention may have a more significant impact on inflammation and iron status. Gong et al. (21) reported that those children who underwent a 1year nutrition-based, comprehensive intervention weight-loss program presented, after this period, lower BMI $z$-scores, and a significant improvement in iron profiles and inflammatory markers IL-6 and CRP, when compared with the control group. As already mentioned, hepcidin levels were not evaluated in this study.

We are aware that our study presents some limitations. Nutritional counseling was provided to all participants; however, we did not administer questionnaires on alimentary habits, which might be a confounding factor in our analyses, even though, and as stated before, inadequate iron ingestion per se does not explain the relationship between obesity and iron deficiency $(7,30,31)$. Considering that the groups were self-selected and a dietary analysis was not conducted, the observed changes may not be due to the PE program alone.

The small number of participants in both groups, particularly in the control group, may have diminished the ability to detect significant alterations in other variables, or in their correlations.

We analyzed overweight and obese participants together, and this may have masked some modifications associated with obesity, even though the control and PE groups were equilibrated for body fat mass and BMI $z$-score at baseline (T1), and included a similar number of obese patients (90\% and $80 \%$ in control and PE groups, respectively; $P=0.222$ ). Besides, a control group of lean children and 
adolescents was not analyzed, which partially limits the discussion of results found at baseline; nonetheless, CRP values are substantially higher than those reported in nonobese controls in a previous work from our group performed in a Portuguese population (median of CRP $=0.25 \mathrm{mg} / \mathrm{l}$ ) (37). Furthermore, the observed correlations between markers of inflammation and of iron metabolism strengthen the association of obesity with low-grade inflammation that triggers the production of hepcidin, compromising iron metabolism. Finally, we cannot exclude the existence (and influence) of sporadic physical activity/exercise outside the intervention program (e.g. at home, after school, and during weekends), which was not reported to our team. Actually, the WHO recommends that "children and youth aged 5-17 should accumulate at least $60 \mathrm{~min}$ of moderate to vigorous-intensity physical activity daily" (25), but we could control only the 5 school days. Nevertheless, those under other known nutritional or PE (structured) programs were excluded from the present study.

In summary, our data show that increasing adiposity in the pediatric population is associated with increasing IL-6, which stimulates hepcidin synthesis, leading to functional iron deficiency due to inhibition of iron absorption and mobilization from iron stores. Moreover, we showed that an 8-monthlong intervention at school allowed a reduction in BMI $z$-score and an improvement in inflammation, namely in IL-6, which causes a reduction in hepcidin levels and in disturbances in iron status.

\section{ACKNOWLEDGMENTS}

This work was funded by European Regional Development Fund (FEDER, Europe) funds through the Operational Competitiveness Programme (COMPETE, Portugal), and by National Funds through Fundação para a Ciência e a Tecnologia (FCT, Portugal) under the project FCOMP-01-0124FEDER-028613 (PTDC/DTP-DES/0393/2012).

Disclosure: The authors declare no conflict of interest.

\section{REFERENCES}

1. McLean E, Cogswell M, Egli I, Wojdyla D, de Benoist B. Worldwide prevalence of anaemia, WHO vitamin and mineral nutrition information system, 1993-2005. Public Health Nutr 2009;12:444-54.

2. Nead KG, Halterman JS, Kaczorowski JM, Auinger P, Weitzman M. Overweight children and adolescents: a risk group for iron deficiency. Pediatrics 2004;114:104-8.

3. Yanoff LB, Menzie CM, Denkinger B, et al. Inflammation and iron deficiency in the hypoferremia of obesity. Int J Obes 2007;31:1412-9.

4. Lecube A, Carrera A, Losada E, Hernandez C, Simo R, Mesa J. Iron deficiency in obese postmenopausal women. Obesity 2006;14: 1724-30.

5. Zimmermann MB, Zeder C, Muthayya S, et al. Adiposity in women and children from transition countries predicts decreased iron absorption, iron deficiency and a reduced response to iron fortification. Int J Obes 2008;32:1098-4.

6. Pinhas-Hamiel O, Newfield RS, Koren I, Agmon A, Lilos P, Phillip M. Greater prevalence of iron deficiency in overweight and obese children and adolescents. Int J Obes Relat Metab Disord 2003;27:416-8.

7. Tussing-Humphreys LM, Liang H, Nemeth E, Freels S, Braunschweig CA. Excess adiposity, inflammation, and iron-deficiency in female adolescents. J Am Diet Assoc 2009;109:297-302.
8. Tussing-Humphreys LM, Nemeth E, Fantuzzi G, et al. Elevated systemic hepcidin and iron depletion in obese premenopausal females. Obesity 2010;18:1449-56.

9. Ausk KJ, Ioannou GN. Is obesity associated with anemia of chronic disease? A population-based study. Obesity 2008;16:2356-61.

10. Coimbra S, Catarino C, Santos-Silva A. The role of adipocytes in the modulation of iron metabolism in obesity. Obes Rev 2013;14:771-9.

11. Anty R, Dahman M, Iannelli A, et al. Bariatric surgery can correct iron depletion in morbidly obese women: a link with chronic inflammation. Obes Surg 2008;18:709-14.

12. Piperno A, Mariani R, Trombini P, Girelli D. Hepcidin modulation in human diseases: from research to clinic. World J Gastroenterol 2009;15: $538-1$.

13. Koorts AM, Viljoen M. Ferritin and ferritin isoforms I: structure-function relationships, synthesis, degradation and secretion. Arch Physiol Biochem 2007;113:30-54.

14. Wrighting DM, Andrews NC. Interleukin-6 induces hepcidin expression through STAT3. Blood 2006;108:3204-09.

15. Verga Falzacappa MV, Vujic Spasic M, Kessler R, Stolte J, Hentze MW, Muckenthaler MU. STAT3 mediates hepatic hepcidin expression and its inflammatory stimulation. Blood 2007;109:353-8.

16. Nemeth E, Rivera S, Gabayan V, et al. IL-6 mediates hypoferremia of inflammation by inducing the synthesis of the iron regulatory hormone hepcidin. J Clin Invest 2004;113:1271-6.

17. Wish JB. Assessing iron status: beyond serum ferritin and transferrin saturation. Clin J Am Soc Nephrol 2006;1(Suppl 1): S4-8.

18. Baumgartner J, Smuts CM, Aeberli I, Malan L, Tjalsma H, Zimmermann MB. Overweight impairs efficacy of iron supplementation in iron-deficient South African children: a randomized controlled intervention. Int J Obes) 2013;37: 24-30.

19. Nascimento H, Costa E, Rocha S, et al. Adiponectin and markers of metabolic syndrome in obese children and adolescents: impact of 8-mo regular physical exercise program. Pediatr Res 2014;76:159-65.

20. Amato A, Santoro N, Calabro P, et al. Effect of body mass index reduction on serum hepcidin levels and iron status in obese children. Int J Obes 2010;34:1772-4.

21. Gong L, Yuan F, Teng J, et al. Weight loss, inflammatory markers, and improvements of iron status in overweight and obese children. J Pediatr 2014;164:795-800 e2.

22. Nascimento H, Alves AI, Medeiros AF, et al. Impact of a school-based intervention protocol - ACORDA Project - on adipokines in an overweight and obese pediatric population. Pediatr Exerc Sci 2016;28:407-16.

23. de Onis M, Onyango AW, Borghi E, Siyam A, Nishida C, Siekmann J. Development of a WHO growth reference for school-aged children and adolescents. Bull World Health Organ 2007;85:660-7.

24. Tanner JM. Normal growth and techniques of growth assessment. Clin Endocrinol Metab 1986;15:411-51.

25. WHO. Global Recommendations on Physical Activity for Health. World Health Organization: Geneva, Switzerland, 2010.

26. Janssen I. Guidelines for physical activity in children and young people. Appl Physiol Nutr Metab 2007;32 (Suppl 2F): S122-35.

27. Strong WB, Malina RM, Blimkie CJ, et al. Evidence based physical activity for school-age youth. J Pediatr 2005;146:732-7.

28. Grandone A, Marzuillo P, Perrone L, Del Giudice EM. Iron metabolism dysregulation and cognitive dysfunction in pediatric obesity: is there a connection? Nutrients 2015;7:9163-70.

29. Hamza RT, Hamed AI, Kharshoum RR. Iron homeostasis and serum hepcidin-25 levels in obese children and adolescents: relation to body mass index. Horm Res Paediatr 2013;80:11-7.

30. Aeberli I, Hurrell RF, Zimmermann MB. Overweight children have higher circulating hepcidin concentrations and lower iron status but have dietary iron intakes and bioavailability comparable with normal weight children. Int J Obes 2009;33:1111-7.

31. del Giudice EM, Santoro N, Amato A, et al. Hepcidin in obese children as a potential mediator of the association between obesity and iron deficiency. J Clin Endocrinol Metab 2009;94:5102-7. 


\section{Articles | Coimbra et al.}

32. Nicolas G, Chauvet C, Viatte L, et al. The gene encoding the iron regulatory peptide hepcidin is regulated by anemia, hypoxia, and inflammation. J Clin Invest 2002;110:1037-44.

33. Tussing-Humphreys L, Pusatcioglu C, Nemeth E, Braunschweig C. Rethinking iron regulation and assessment in iron deficiency, anemia of chronic disease, and obesity: introducing hepcidin. J Acad Nutr Diet 2012;112:391-400.

34. Bekri S, Gual P, Anty R, et al. Increased adipose tissue expression of hepcidin in severe obesity is independent from diabetes and NASH. Gastroenterology 2006;131:788-96.
35. Cepeda-Lopez AC, Allende-Labastida J, Melse-Boonstra A, et al. The effects of fat loss after bariatric surgery on inflammation, serum hepcidin, and iron absorption: a prospective 6-mo iron stable isotope study. Am J Clin Nutr 2016;104:1030-8.

36. Tussing-Humphreys LM, Nemeth E, Fantuzzi G, et al. Decreased serum hepcidin and improved functional iron status 6 months after restrictive bariatric surgery. Obesity 2010;18:2010-6.

37. Nascimento H, Costa E, Rocha-Pereira P, et al. Cardiovascular risk factors in portuguese obese children and adolescents: impact of small reductions in body mass index imposed by lifestyle modifications. Open Biochem J 2012;6:43-50. 